\title{
Preparing for ENDF/B-VIII
}

\author{
David Brown ${ }^{\mathrm{a}}$ and Cross Section Evaluation Working Group (CSEWG) \\ Brookhaven National Laboratory, National Nuclear Data Center, Upton NY, USA
}

\begin{abstract}
Although the next major release of the ENDF/B library is not due until the 2017-2018 time frame, ENDF/B-VIII is already positioned to become the most important release of the library in some time. ENDF/B-VIII will be built around the Neutron Reaction Standards as well as the ${ }^{1} \mathrm{H},{ }^{16} \mathrm{O},{ }^{56} \mathrm{Fe},{ }^{235} \mathrm{U},{ }^{238} \mathrm{U}$ and ${ }^{239} \mathrm{Pu}$ evaluations developed as part of the Coordinated International Evaluation Library Organization (CIELO) pilot project. In this contribution, we summarize these improvements as well as the many other improvements to ENDF that have already been made or are scheduled to be made in the next year. Improvements already included in the ENDF/B-VIII beta releases:
\end{abstract}

- Aggressive use of the flexible and physically correct $\mathrm{LRF}=7$ resolved resonance format in 12 updated evaluations $\left({ }^{35,37} \mathrm{Cl},{ }^{40} \mathrm{Ca},{ }^{54,56,57} \mathrm{Fe},{ }^{63,65} \mathrm{Cu}\right.$ and $\left.{ }^{182,183,184,186} \mathrm{~W}\right)$

- Thermal capture gammas from the EGAF project $\left({ }^{6,7} \mathrm{Li},{ }^{11} \mathrm{~B},{ }^{19} \mathrm{~F},{ }^{23} \mathrm{Na},{ }^{27} \mathrm{Al},{ }^{28} \mathrm{Si},{ }^{35,37} \mathrm{Cl}\right)$

- Thermal Scattering Law evaluations from NCSU ( $\alpha$ and $\beta$ phase $\mathrm{SiO}_{2}, \mathrm{SiC}$, lucite, $\mathrm{BeO}$, and polyethylene) and from the CAB-CNL collaboration (heavy and light water)

- Many new evaluations in the neutron sublibrary $\left(\mathrm{n},{ }^{12,13} \mathrm{C},{ }^{40} \mathrm{Ar},{ }^{54,57,58} \mathrm{Fe},{ }^{58,59,60,61,62,64} \mathrm{Ni},{ }^{63,65} \mathrm{Cu},{ }^{73} \mathrm{As},{ }^{120} \mathrm{Sn},{ }^{236 m 1} \mathrm{~Np}\right)$

- Inclusion of Red Cullen's EPICS2014 library, updating the photo-atomic, electron and atomic-relaxation sublibraries.

Many improvements are planned in the next year including new evaluations such as charged particle evaluations translated from LLNL's ECPL. In addition to these major changes, ENDF/B-VIII will be the first official library released simultaneously in the legacy ENDF-6 and the newly developed Generalized Nuclear Data (GND) formats.

\section{Status of ENDF/B-VIII}

ENDF/B is the United States' most important data library for nuclear applications and is used in nearly all particle transport codes. As a result, ENDF/B-VII.1 (the previous release, published in 2010), contained 423 evaluations in the neutron sublibrary and has been cited in literature 913 times as of September 2016 [1]. The last major release, ENDF/B-VII.0, was cited an astounding 1469 times [2]. With this history, the Cross Section Evaluation Working Group (CSEWG) is preparing another major release: ENDF/B-VIII. The core of this library are the CIELO evaluations [3] described below as well as the host of other improvements and additions also listed below.

We have made three ENDF/B-VIII beta releases before ND2016, the last in August 2016. With each release, the library has improved dramatically. We anticipate several more beta releases before the end of 2017.

The complete list of changes and additions are available at the NNDC GForge site (https://ndclx4.bnl. gov/gf/project/endf). The $\beta 2$ release is available at https://ndclx4.bnl.gov/gf/project/endf/ scmsvn/?action=browse\&path=\%2Ftags $\% 2 \mathrm{FENDF}-\mathrm{B}-$ VIII.beta $2 \% 2 \mathrm{~F}$ for the subversion repository and https://ndclx4.bnl.gov/gf/project/endf/frs/ for tarballs of the data.
In this contribution, we will highlight some of the major features in ENDF/B-VIII and direct readers to some of the other CSEWG contributions to this conference.

\section{Neutron standards and CIELO evaluations}

Every major release of the ENDF/B library coincides with new releases of the Neutron Standards evaluations and ENDF/B-VIII is no exception. The Neutron Standards Project is a long standing IAEA Nuclear Data Section Data Development Project to develop reference neutron cross sections. The new Neutron Standards evaluations will be detailed in A. Carlson contribution R181: "A new evaluation of the neutron data standards".

With the long-term success of the Standards project the CIELO pilot project was created in order to deliver standards-quality full evaluations to the data community for inclusion in regional libraries such as ENDF/B. Given the significant contribution of US based scientists to CIELO, it is no surprise that CSEWG has chosen to include CIELO evaluations in ENDF/B-VIII. The CIELO project is described in M. Chadwick's plenary talk (PL499) and different aspects of the CIELO project from CSEWG members are presented in a series of other contributions to this conference:

S100: R. Capote "New fit of neutron thermal constants for ${ }^{233,235} \mathrm{U},{ }^{239,241} \mathrm{Pu}$ and ${ }^{252} \mathrm{Cf}$ : microscopic

\footnotetext{
a e-mail: dbrown@bnl.gov
}

(C) The Authors, published by EDP Sciences. This is an Open Access article distributed under the terms of the Creative Commons Attribution License 4.0 (http://creativecommons.org/licenses/by/4.0/). 


\section{Ca40(n,tot)}

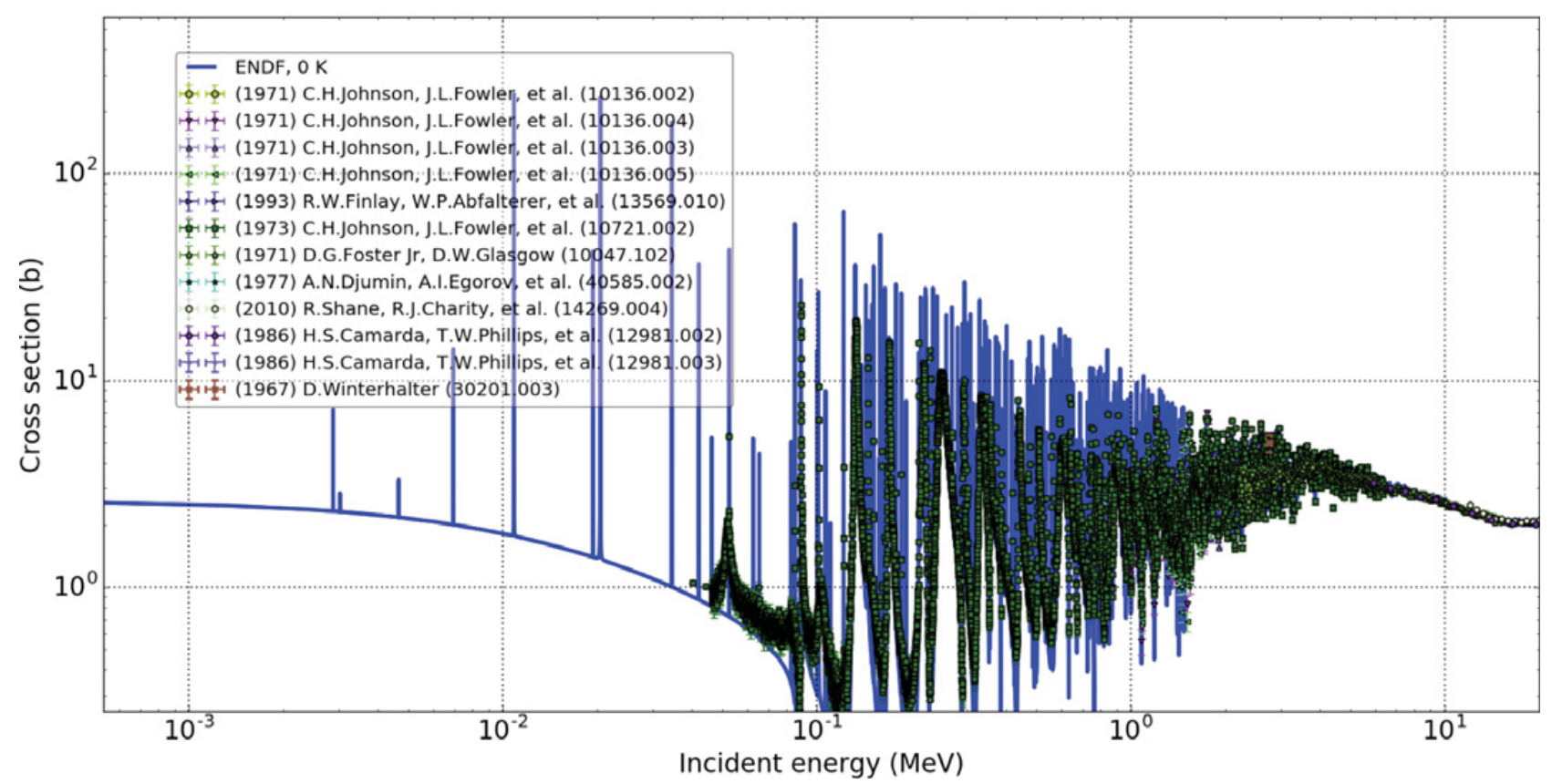

Figure $1 .{ }^{40} \mathrm{Ca}$ (n,tot) cross section from the new ORNL evaluation in ENDF/B-VIII. The new evaluation is given in blue and the world's collection of total reaction data as stored in EXFOR [4] are shown in the symbols.

vs. integral data"; I102: “238 U evaluation and validation of the neutron induced reactions up to $20 \mathrm{MeV}$ "

R095: G. Hale and M. Paris: "Neutron cross sections for carbon and oxygen from new R-matrix analyses of the ${ }^{13,14} \mathrm{C}$ and ${ }^{17} \mathrm{O}$ systems"

R096: M. Herman on the status of the ${ }^{56} \mathrm{Fe}$ evaluation

R106: M. Pigni " $\mathrm{n}+{ }^{235} \mathrm{U}$ Resonance Parameters and Neutrons Multiplicities in the Energy Region below $100 \mathrm{eV}$ ".

\section{Non-CIELO evaluations}

Clearly ENDF/B-VIII is more than the Standards and CIELO evaluations. In the three beta releases so far CSEWG has added/updated many evaluations to the neutron sublibrary: $\mathrm{n}$ (LANL), ${ }^{18} \mathrm{O}$ (RUSFOND), ${ }^{40} \mathrm{Ar}$ (LANL), ${ }^{40} \mathrm{Ca}$ (ORNL), ${ }^{54,57,58} \mathrm{Fe}$ (BNL), ${ }^{73} \mathrm{As}$ (LANL), $\mathrm{Yb}$, Os, holes in the isotope chains for Dy, Hf (JENDL-

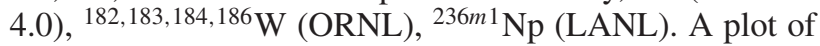
the new ${ }^{40} \mathrm{Ca}$ evaluation is shown in Fig. 1. In addition, primary gammas from EGAF were added to ${ }^{6,7} \mathrm{Li},{ }^{11} \mathrm{~B}$, ${ }^{23} \mathrm{Na},{ }^{27} \mathrm{Al},{ }^{28} \mathrm{Si}$, and ${ }^{35,37} \mathrm{Cl}$.

As neutrons are moderated to lower energies, scattering eventually can not be described by neutrons interacting with single nuclei. Instead neutrons scatter off macroscopic collections of nuclei so one uses the thermal neutron scattering sublibrary. A. Hawari's group at North Carolina State University has been especially prolific at developing thermal neutron scattering evaluations, having evaluated polyethylene, lucite, $\mathrm{BeO}(\mathrm{OinBeO}$ and BeinBeO), $\mathrm{SiC}$ (SiinSiC and $\mathrm{CinSiC}$ ), and $\alpha$ and $\beta$ phases of $\mathrm{SiO}_{2}$, as discussed in Hawari's contribution I069. Plots of the polyethylene evaluation are shown in Fig. 2. Additionally, a collaboration between Canadian National Laboratory and Centro Atómico Bariloche (Argentina) has developed evaluations for light and heavy water as described in contribution 1060 by J.I. Marquez Damian.

CSEWG has adopted the electro/photo-atomic and atomic- relaxation data from D.E. Cullen's EPICS2014 library [7] and added beta branchings to 24 isotopes in the decay sublibrary.

\section{Theoretical and methodological developments}

The push to develop standards level evaluations for CIELO has led CSEWG to adapt and improve models used in evaluations and the evaluation methodology. Contribution S503 from T. Kawano describes an inter-comparison of Hauser-Feshbach model codes aimed at better actinide evaluations. In addition to this comparison, there have been efforts to include the Englebrecht-Weidenmueller transformation [8] in reaction models and to tighten the connections between Hauser Feshbach and R-matrix approaches:

I329: T. Kawano: "Advances in nuclear reaction calculations by incorporating information from nuclear mean-field theories"

R183: D. Brown: "An analytic approach to probability tables for the unresolved resonance region".

In addition to the improvements to the fast region modeling, there have been advances in R-matrix codes:

S354: M. Pigni "Early Applications of the R-matrix SAMMY Code for Charged-particle Induced Reactions"

R259: Limited inclusion of integral data into evaluation process Vladimir Sobes "Application Of The SAMINT Methodology To The New CrossSection Evaluations of ${ }^{63} \mathrm{Cu}$ and ${ }^{65} \mathrm{Cu}$ ". 

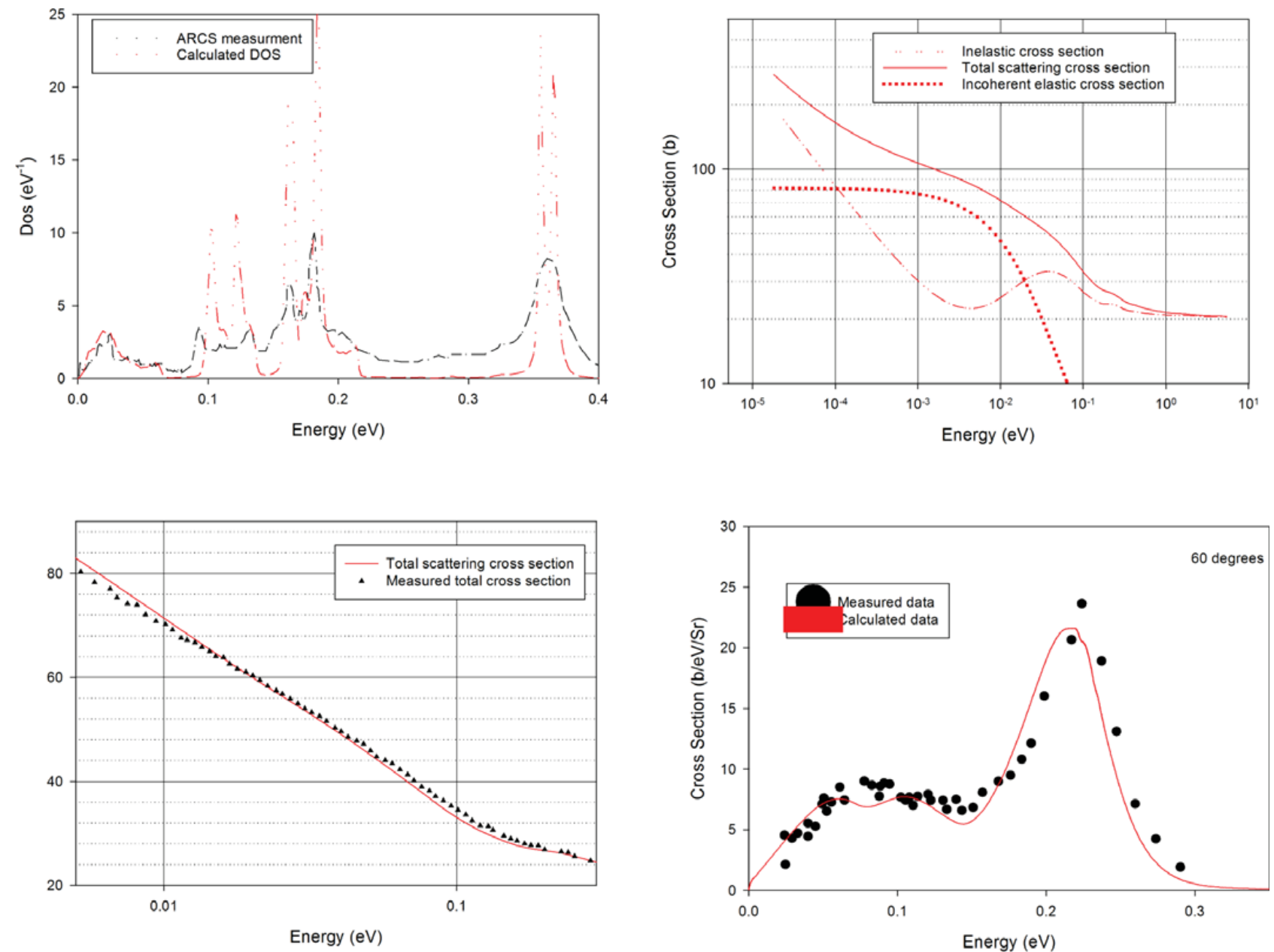

Figure 2. The polyethylene thermal scattering law evaluation from North Carolina State University in ENDF/B-VIII. In the upper left hand panel, we show the density of states used in the polyethylene evaluation along with the data from Lavelle, et al. [5]. In the upper right hand panel, we compare the total scattering, inelastic scattering and the incoherent elastic scattering cross sections. In the lower left panel, we show the total scattering cross section compared the data taken from EXFOR [4]. In the lower right panel, we show the incoherent elastic cross section compared to the data from EXFOR [4]. This figure taken from Ref. [6].

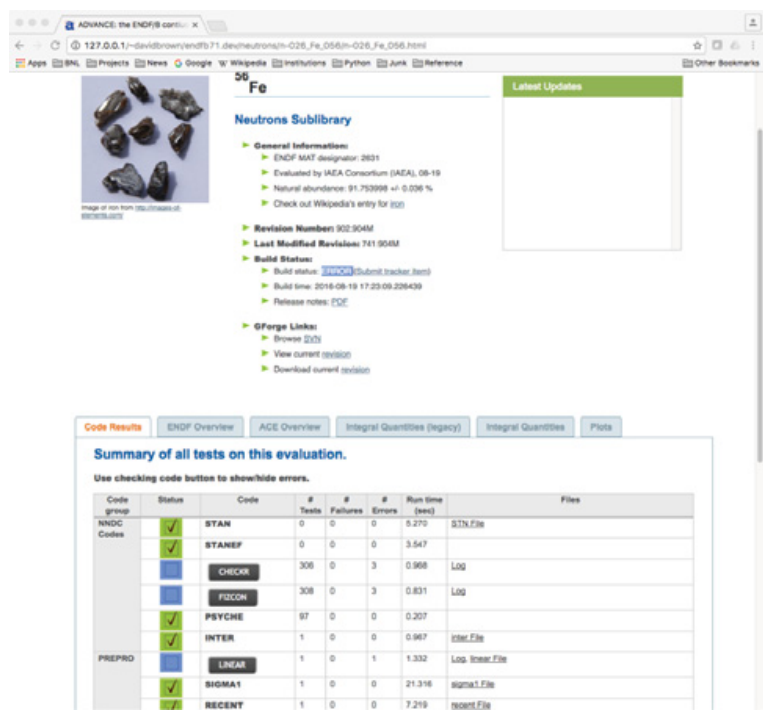

Figure 3. Screenshot of a typical ADVANCE build report. Successful builds are denoted with the green checkmark, builds with errors are given the blue box and failed builds are given a red "bomb". Fortunately there are no failures in this build. Error messages can be reviewed using the black button for each build.

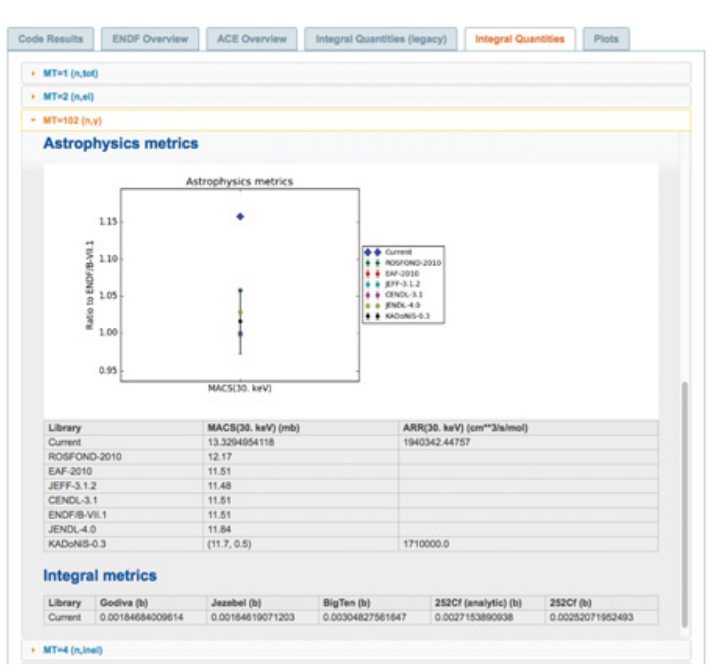

Figure 4. Screenshot of the new integral quantity report being phased into the ADVANCE QA reports. Metrics such as the $30 \mathrm{keV}$ Maxwellian Averaged Cross Section (MACS) are compared to results from the KADoNiS library [9] and a variety of other databases. Comparison data was taken from Ref. [10]. 


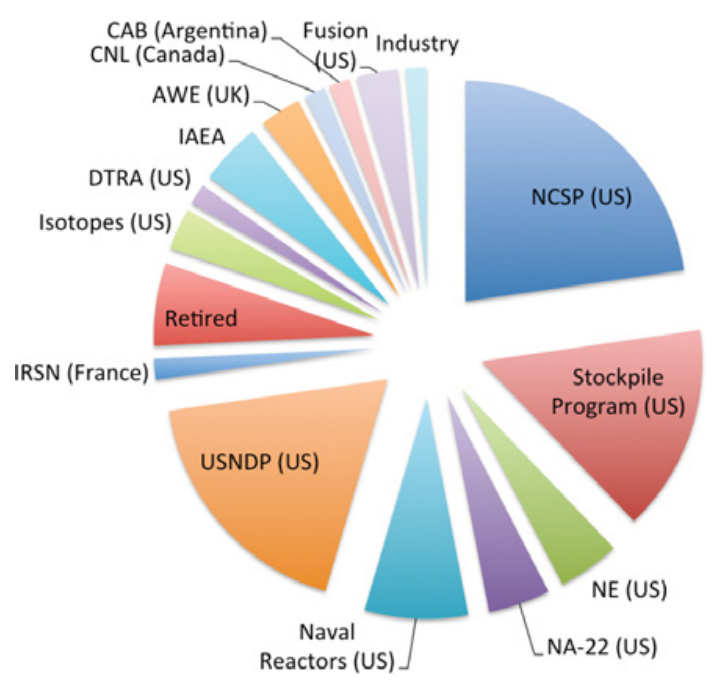

Figure 5. Breakdown of participation at the November 2015 Cross Section Evaluation Working Group meeting.

\section{Testing and quality assurance}

The improvements and additions to ENDF/B are only useful if there a rigorous quality assurance program to ensure the correctness and usability of the data. CSEWG has traditionally adopted a Phase I/II system where Phase I testing ensures the basic format and physics correctness of evaluations and Phase II testing involves testing the new evaluations in integral benchmarks. Nowadays, the entire Phase I testing has been automated using the ADVANCE continuous integration system. ADVANCE runs each evaluation through a battery of tests and through the PREPRO, NJOY and FUDGE processing codes to ensure that users can process the data (see the screenshot in Fig. 3). ADVANCE has recently been adapted to include a new version of the INTER code (see the screenshot in Fig. 4) and we are in the process of adding automated benchmarking to assist at Phase II testing. Complete ADVANCE build reports are available at http://www.nndc.bnl.gov/endf/b7.dev/qa/index. html.

After the testing in ADVANCE, the ENDF files undergo rigorous Phase II testing. In this conference, several contributions describe the results of integral benchmark testing of ENDF/B-VIII beta releases:
1094: A. Kahler "Criticality data testing of CIELO evaluated nuclear data files"

R311: M.-A. Descalle "Criticality Benchmark Simulations with ENDF/B- VIII.beta Structural Material Evaluations in GND Format"

R230: M. Pigni "Validation of W Cross Sections in the Neutron Energy Region up to $100 \mathrm{keV"}$ ".

\section{About CSEWG}

The ENDF/B library is the main product of the Cross Section Evaluation Working Group (CSEWG). CSEWG is a long running yet very informal collaboration now in its $50^{\text {th }}$ year. CSEWG encourages collaboration with experimentalists, theorists, evaluators, data testers and data testers worldwide as evidenced by the graphic in Fig. 5 .

This work is supported by the DOE Office of Nuclear Physics under Contract No. DE-AC02-98CH10946

\section{References}

[1] M.B. Chadwick, M. Herman, P.P. Obložinský, et al., Nucl. Data Sheets 112, 2887-2996 (2011)

[2] M.B. Chadwick, P.P. Obložinský, M. Herman at al., Nucl. Data Sheets 107, 2931-3060 (2006)

[3] M.B. Chadwick, E. Dupont, E. Bauge, et al. Nucl. Data Sheets 118, 1-25 (2014)

[4] N. Otuka, E. Dupont, V. Semkova, et al., Nucl. Data Sheets 120, 272 (2014)

[5] C.M. Lavelle, C.-Y. Liu, M.B. Stone, Nucl. Inst. Meth. A 711, 166 (2013)

[6] A.I. Hawari, OECD/NEA Meeting: WPEC SG42 "Thermal Scattering Kernel $S(\alpha, \beta)$ : Measurement, Evaluation and Application" May 10-11, 2016, Paris, France

[7] D.E. Cullen, IAEA Report IAEA-NDS-218 (2014)

[8] T. Kawano, R. Capote, S. Hilaire, P. Chau Huu-Tai, Phys. Rev. C 94, 014612 (2016)

[9] I. Dillmann, R. Plag, F. Käppeler, T. Rauscher Submitted as proceeding of the workshop "EFNUDAT Fast Neutrons - scientific workshop on neutron measurements, theory \& applications" held on April 28-30 2009 at Geel, Belgium (2009)

[10] B. Pritychenko Nucl. Data Sheets 123, 119-123 (2015) 